\title{
Design of Multimedia Classroom Control System Applying PLC and KingView
}

\author{
Y.C. CHEN \& W.G. LI \& Q.L. YANG \& L.L. HUANG \\ Faculty of Mechanical \& Electrical Engineering, Kunming University of Science \& Technology, Jingming \\ South Road, Kunming, Yunan, 650504, P.R. China
}

\begin{abstract}
Because of the wide application of multimedia in classroom, many problems have been appeared. This paper presents the design of control system for multimedia classroom using PLC and KingView, the KingView soft is applied to design the multimedia monitoring interface, and the PLC is used to set up the hardware core, and to implement the control task. This paper explains the design of KingView monitoring system and the main structure of monitoring unit of PLC in detail. Our simulated experiment shows that the proposed design approach is correct, and the proposed approach can realizes the integration of supervision and control technology, and I believe this monitoring system will obtain a widely application in many fields.
\end{abstract}

KEYWORD: PLC; ladder diagram; multimedia classroom; KingView;

\section{INTRODUCTION}

The traditional multimedia classroom needs the use and maintenance of multimedia classroom staffs, and classroom staffs also help the teacher to solve the device fault. Multimedia classroom staffs often help teachers to turn on the control system before class and to turn off the control system after class, they also help to deal with the emergencies which take place in the course, and finally, they need to lock the door while all the classes are over. These works increase the load of classroom staff, and reduce the work efficiency. With more and more application of multimedia in the modernization of classroom, many problems have also been appeared, in this paper, a design approach of control system of multimedia classroom is presented to solve above problems, the PLC (programmable logic controller) and the configuration software KingView are used to structure the control system of multimedia classroom, which will reduce many complicated works of the teaching staffs.

Intelligential requirements of multimedia classroom are increasingly high with the development of science and technology. Therefore, in recent years, renewal and transformation for multimedia classroom equipment become more and more quickly. Improvement of the efficiency and quality of teaching is more and more important. In this paper, the multimedia classroom control system is designed by PLC (Mitsubishi FX2N). At present, many researchers have proposed a lots of control approaches for multimedia classroom control system applying PLC technique. The design of intelligent lighting control system for classroom was presented by Li etc. [1], the control system design of classroom light based on MCU was proposed by $\mathrm{Li}$ and Zhao [2], the design of step motor control system based on PLC was proposed by $\mathrm{Li}$ and $\mathrm{Li}$ [3], the experimental system of controlling stepping motor based on PLC was proposed by He etc. [4].

\section{DESIGN OF MONITORING SYSTEM FOR MULTIMEDIA CLASSROOM}

\subsection{The controlled object of multimedia classroom}

The controlled object for multimedia equipment comprises the projector, digital video presenter, digital control system, screen, power amplifier, sound box, control desk, application software, and auxiliary materials. Generally multimedia classroom is mainly composed of the following three parts:

(1) Multimedia projector

Multimedia projector is the most expensive and the most important equipment in multimedia presentation classroom; it is connected with the computer system, the video output system and digital video showcase. The video, digital signal will be outputted and appeared on the big screen.

(2) The multimedia computer 
The multimedia computer is the core of demonstration system; teaching software should be controlled by it, and decides the demonstration effect and the quality in a great degree.

(3) The central control system

Central control system uses the method of system integration; integrate the entire equipment operation of multimedia demonstration classroom in one platform, all the equipment operation can be completed in the platform.

\subsection{Control function of multimedia classroom}

The main control functions in multimedia classroom includes: (1) The power turning on and the power turning off; (2) The running and the stopping of the projector; (3) The falling and the rising of the screen;

(4) The sound turning on and the sound turning off;

(5) The opening and the closing of classroom door;

(6) The lamp turning on and the lamp turning off; (7) The opening and the closing of classroom curtain.

\subsection{Design of the control ladder diagram for multimedia classroom}

The steps of the design of the control ladder diagram is as follows: [5]

Firstly, open the "GX DEVELOPER" soft, which is the programmable tool for ladder diagram design.

Secondly, the ladder diagram is drawn in "GX DEVELOPER" soft by computer, and then is compiled and saved in the computer after the ladder diagram design is finished.

Thirdly, implement the running simulation to validate the correctness of the drawn control ladder diagram in "GX Simulator 6" soft.

Figure. 1 shows the control ladder diagram for screen falling that is drawn by "GX DEVELOPER" soft. The description of the control ladder diagram for screen falling is as follows:

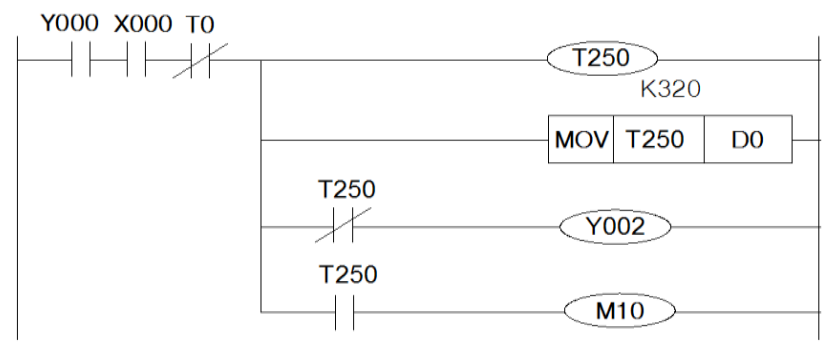

Figure1. The control ladder diagram of screen falling

(1) The motor is controlled by a normally closed timer T251, after 32 seconds the motor stops rotating automatically and the screen stop down;

(2) Screen position is controlled by a DO timer; it beams T250 data to DO, simulate the rise and the fall of screen on interface of KingView.
(3) Selection screen fall time. Through the study of multimedia classroom, the reference of screen standard specification, and the requirements of KingView, 32 second is regard as the requirements time in this projection control design.

(4) Selection normally closed contactor. Consider the T250 of the timer will re-timing after 32 seconds, add a non-cumulative timer T0 to guarantee power supply, timer T250 will disconnected after 32 seconds and cannot continue timing.

\subsection{Design of multimedia classroom monitoring system based on KingView}

This design of monitoring system is drawn by KingView, it provides a visual monitor screen for testing, and real-time site monitoring. Moreover, it can make full use of Windows graphic editing function, to constitute a monitoring screen easily, under normal circumstances, the establishment of a KingView application engineering can be broadly divided into the following steps: [6][7]

(1)Create a new project

Select the menu "filelnew construction" dialog box; click "next" to choose paths for the project.

(2)Definition of external equipment

KingView need to definite equipment and programs before programming, and require the data be exchanged between the external devices. External device comprises a lower machine (PLC, instrumentation, module, inverter etc.); they generally exchange data through the serial port and PC. Other Windows applications, the general data exchange between them is done by DDE. The external devices also include other computers on the network.

KingView can transform data with them through $\mathrm{I} / \mathrm{O}$ variables only after the external equipment is defined. KingView designs "the device configuration wizard", for the convenience of definition of the external equipment; KingView guides users to complete the equipment connection step by step.

Click "outline equipment" under the OPC server with the left mouse button in the display area of the project browser directory, the display area will appear "new" icon in the directory. And then click "connection with OPC server"; the external equipment setup is completed.

(3)Definition of variable data

The collection of variables is vividly called "the data dictionary in KingView, data dictionary records the detailed information of the variable data that all the users can use. And "data dictionary" is located in the drop-down list of "database" in KingView project browser. The variables obtained for monitoring system of the multimedia classroom are listed in the Table.1. 
Table 1 Variable quantity in KingVeiw

\begin{tabular}{|c|c|c|c|c|}
\hline Variable name & Device name & Register name & Data type & Variable type \\
\hline Screen falling button & Mitsubishi.XOPC.4 & Dev01.X.X000 & Bit & I/O Discrete \\
\hline Screen falling indicator & Mitsubishi.MXOPC.4 & Dev01.Y.Y002 & Bit & I/O Discrete \\
\hline Screen rising indicator & Mitsubishi.XOPC.4 & Dev01.Y.Y003 & Bit & I/O Discrete \\
\hline Screen rising button & Mitsubishi.XOPC.4 & Dev01.X.X001 & Bit & I/O Discrete \\
\hline Projector turn on & Mitsubishi.XOPC.4 & Dev01.M.M1 & Bit & I/O Discrete \\
\hline Power on & Mitsubishi.XOPC.4 & Dev01.M.M0 & Bit & I/O Discrete \\
\hline Power indicator & Mitsubishi.XOPC.4 & Dev01.Y.Y000 & Bit & I/O Discrete \\
\hline Projector display button & Mitsubishi.XOPC.4 & Dev01.Y.Y001 & Bit & I/O Discrete \\
\hline Projector turn off & Mitsubishi.XOPC.4 & Dev01.M.M2 & Bit & I/O Discrete \\
\hline Door opening & Mitsubishi.XOPC.4 & Dev01.M.M5 & Bit & I/O Discrete \\
\hline Door closing & Mitsubishi.XOPC.4 & Dev01.M.M6 & Bit & I/O Discrete \\
\hline Power off & Mitsubishi.XOPC.4 & Dev01.M.M14 & Bit & I/O Discrete \\
\hline Sound turn on & Mitsubishi.XOPC.4 & Dev01.M.M3 & Bit & I/O Discrete \\
\hline Sound turn off & Mitsubishi.XOPC.4 & Dev01.M.M4 & Bit & I/O Discrete \\
\hline Door control indicator & Mitsubishi.XOPC.4 & Dev01.Y.Y005 & Bit & I/O Discrete \\
\hline Sound control indicator & Mitsubishi.XOPC.4 & Dev01.Y.Y004 & Bit & I/O Discrete \\
\hline Light control indicator & Mitsubishi.XOPC.4 & Dev01.Y.Y006 & Bit & I/O Discrete \\
\hline Light turn on & Mitsubishi.XOPC.4 & Dev01.M.M7 & Bit & I/O Discrete \\
\hline Light turn off & Mitsubishi.XOPC.4 & Dev01.M.M8 & Bit & I/O Discrete \\
\hline Curtain rising & Mitsubishi.XOPC.4 & Dev01.M.M12 & Bit & I/O Discrete \\
\hline Curtain falling & Mitsubishi.XOPC.4 & Dev01.M.M13 & Bit & I/O Discrete \\
\hline Curtain control indicator & Mitsubishi.XOPC.4 & Dev01.Y.Y007 & Bit & I/O Discrete \\
\hline Position of screen rising & Mitsubishi.XOPC.4 & Dev01.d.d1 & short & I/O Integer \\
\hline Position of screen falling & Mitsubishi.XOPC.4 & Dev01.d.d0 & short & I/O Integer \\
\hline
\end{tabular}

(4)Produce graphic image

The user can build screen graphical interface easily in KingView by using object-oriented programming technology. The user can use the graphical object provided by the system to complete the creation of picture that is like building blocks, at the same time, KingView supports graphics object replication between screens, and can use the previous development results repeatedly.

There is no limit to the number of the pictures that can be established for each project, and interrelated static or dynamic graphical objects in each picture will be generated after entering the KingView development system. These pictures are composed by the graphics objects that have rich types of KingView. These pictures can paint screen, projector, doors, lights, sound and curtain. For example, opens the "Gallery" option to select the icon, "button" option to design while making the screen control button.

(5)The establishment of the connection between animations

The so-called "animation connection" is to establish the corresponding relations between picture element and variable data. Pictures made by KingView are static, the introduction of "animation connection" is a breakthrough in the design of manmachine interface, it liberates the engineering personnel from the repetitive graphics programming, provides standard industry control graphics interface for engineers, and enhances the graphical interface function by the connection of a programmable command language. [8]

\section{EXPERIMENT}

To validate the correctness and stability of this design approach, we do lots of simulated experiments. Before the simulated experiments, the connection settings between the PLC and KingView need to be firstly done, and the right COM port need to be chosen to connect the normal communication.

Figure. 2 shows the actual connection diagram between PLC and KingView soft, which is used to validate the correctness of the proposed approach. Figure. 3 shows the simulated experiment results of the screen is falling down, and the monitoring interface is drawn by KingView, from Figure.3, we 
can obtain that the screen is falling down, but do not reach the lowest position. Figure. 4 shows the simulated experiment results of control for sound, lamps, curtains, and doors, which denotes these devices are turned on, and the monitoring interface is drawn by KingView, from Figure.4, we can obtain the lamp is turned on (the top of classroom is bright), the curtain is pulled down (the right of classroom), and the door is opened (the northwest of classroom is opened). Figure. 4 shows that the proposed approach is completely correct.

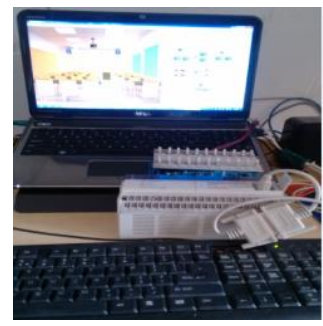

Figure 2. The actual connection diagram between PLC and KingView

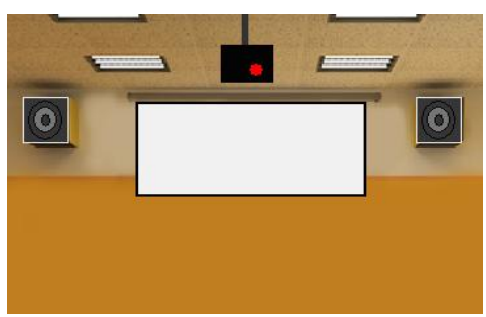

Figure 3. The simulated experiment result for the screen falling.

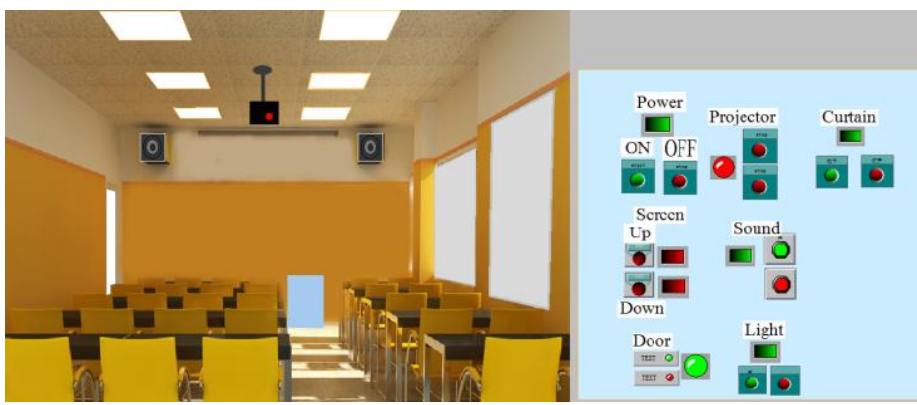

Figure 4. The simulated experiment result while the sound, lamps, curtains, and doors are turned on.

\section{CONCLUSIONS}

The multimedia classroom control system combines the techniques of PLC and the configuration software KingView to realize the monitoring and management of multimedia classroom. The proposed control interface is simple, practical, stable and reliable, and the operation is convenient. The applications of this system will save the complicated work of teaching staffs. The teachers can monitor and manage the multimedia teaching system directly. The operation is more intelligent, simple. Therefore, the teachers can only focus on the students, and the multimedia teaching activities is convenient for teachers to improve the teaching quality.

\section{REFERENCES}

[1] Li, Q.H. \& Shang, J.L. \& Zhang, G.B. \& Yang, H.X. 2007. Design of Intelligent Lighting Control System for Classroom. Computer Measurement \& Control in Chinese. 15(8): 1011-1013.

[2] Li, L. \& Zhao, G.K. 2004. The Control System Design of Classroom Light Based on MCU. Machinery \& Electronics in Chinese. (6): 69-70.

[3] Li, W.H. \& Li, G.Q. 2008. Design of Step Motor Control System Based on PLC. Instrumentation Technology in Chinese. (9): 23-25.

[4] He, Y. \& Wu, S.J. \& Guo, X.Y. 2008. Experimental System of Controlling Stepping Motor Based on PLC. Mechanical Management and development in Chinese. 23(5): 72-74.

[5] Han, X.X. 2011. From basic to the practice of PLC and KingView. Beijing: China Mechanical Industry Press.

[6] Jiang, S.H. 2005. Monitoring system of large water plant based on PLC and configuration software. Industrial Control Computer in Chinese, 18(3):69-70.

[7] Cao, H. 2009. Configuration software technology and application. Beijing: china electronics industry press.

[8] Wang, Y.M. 2003. The design and development of configuration software. Xi'an: Xi'an Electronic and Science University press. 